\title{
DYNAMICS OF TYPICAL CONTINUOUS FUNCTIONS
}

\author{
HERVE LEHNING
}

(Communicated by Andrew Bruckner)

\begin{abstract}
S. J. Agronsky, A. M. Bruckner, and M. Laczkovic have studied the behaviour of the sequence $\left(f^{n}(x)\right)$ where $f$ is the typical continuous function from the closed unit interval $I$ into itself and $x$ the typical point of $I$. In particular, they have proved that the typical limit set $\omega(f, x)$ is a Cantor set of Menger-Uryson dimension zero. Using mainly the Tietze extension theorem, we have found a shorter proof of this result which applies to a more general situation. As a matter of fact, we have replaced the closed unit interval by a compact $N$-dimensional manifold and the Menger-Uryson dimension by the Hausdorff one. We have also proved that, for the typical continuous function $f$, the function $x \rightarrow \omega(f, x)$ is continuous at the typical point $x$. It follows that the typical limit set is not a fractal and that, for the typical continuous function $f$, the sequence $\left(f^{n}(x)\right)$ is not chaotic.
\end{abstract}

\section{INTRODUCTION}

From now onward, we set $X$ to denote a compact manifold with boundary, $N$ to denote its dimension, and $d$ to denote a metric on $X$ such that $X$ is a second countable complete metric space (i.e., having a countable basis). If $B$ is a subset of $X$, we shall represent its interior by $\operatorname{Int}(B)$, its closure by $\operatorname{Clos}(B)$, its exterior (i.e., $X-\operatorname{Clos}(B))$ by $\operatorname{Ext}(B)$, its boundary by $\partial B$, and its diameter by $d(B)$.

$K(X)$ will denote the set of compact subsets of $X$ and $d_{K}$ the Hausdorff metric on $K(X)$ (see [5, p. 96]). Thus, $K(X)$ is a metric space.

$C(X)$ will denote the space of continuous functions from $X$ into itself and $d_{\infty}$ the metric of uniform convergence on $C(X)$. Thus, $C(X)$ is a complete metric space. With the metric $\delta=\max \left(d_{\infty}, d\right), C(X) \times X$ is also a complete metric space. If $(f, x) \in C(X) \times X$, we shall denote by $\omega(f, x)$ the limit set (i.e., the set of the limit points) of the sequence $\left(f^{n}(x)\right)$. Thus $\omega$ is a function from $C(X) \times X$ into $K(X)$.

If $E$ is a complete metric space, we shall call residual a subset of $E$ which contains a countable intersection of dense, open subsets. If $P(x)$ is a property, the sentences "for $x$ typical in $E, P(x)$ ", "if $x$ is typical in $E, P(x)$ ", and " $P(x)$ at the typical point $x$ of $E$ " will mean "there is a residual subset $A$ of

Received by the editors November 26, 1991 and, in revised form, October 14, 1992 and September 13, 1993.

1991 Mathematics Subject Classification. Primary 26A18. 
$E$ such that, if $x$ belongs to $A$, then $P(x)$ ". In the same situation, we may also say that " $P(x)$ is typical in $E$ " or " $P(x)$ is typical".

\section{LEMMAS}

The proofs of our theorems rely on two lemmas which mainly use the Tietze extension theorem (in a particular version, see [2, Corollary 1, p. 82]).

Lemma 1. Let $\alpha>0, \beta>0$, and let $E_{\alpha, \beta}$ be the set of those $(f, x) \in C(X) \times X$ such that there is a finite number of subsets $\left(k_{i}\right)_{i}$ verifying:

- $d\left(k_{i}\right)<\beta$ for each $i$

- $\omega(f, x) \subset \bigcup_{i} k_{i}$ and $\sum_{i} d\left(k_{i}\right)^{\alpha}<1$.

The set $E_{\alpha, \beta}$ contains an open, dense set in $C(X) \times X$.

Proof. Let $(f, a) \in C(X) \times X$ and $\varepsilon>0$. Using the compactness of $X$, we construct a finite cover of open sets of diameters strictly less than $\varepsilon$ having a closure homeomorphic to $I^{N}$ where $I$ is the closed unit interval.

Let $p$ be the smallest positive integer such that there are an integer $n<p$ and an element $V$ of the cover containing $f^{p}(a)$ and $f^{n}(a)$. Let $m=p-n$. This property implies that the points $a, f(a), f^{2}(a), \ldots, f^{n+m-1}(a)$ are distinct.

Let $A$ be a closed subset, $B$ and $C$ two open subsets, and $y \notin A$ such that $f(y) \in B$ and $y \in C$. Using the continuity of $f$ at the point $y$, we can construct a compact subset $K$ homeomorphic to $I^{N}$ such that: $K \cap A=\varnothing$, $K \subset C, y \in \operatorname{Int}(K), d(K)<\varepsilon$, and $f(K) \subset B$.

We use this property for $A=\left\{a, f(a), \ldots, f^{n+m-2}(a)\right\}, y=f^{n+m-1}(a)$, $B=V$, and $C=X$, and obtain a compact $K_{n+m-1}$. Using it for $A=$ $\left\{a, f(a), \ldots, f^{n+m-3}(a)\right\} \cup K_{n+m-1}, y=f^{n+m-2}(a), B=\operatorname{Int}\left(K_{n+m-2}\right)$, and $C=X$, we obtain $K_{n+m-2}$. We continue in the same way (with the exception of $C=V$ for $K_{n}$ ). Eventually, we have constructed a sequence of compacts $K_{0}, K_{1}, \ldots, K_{n+m-1}$ homeomorphic to $I^{N}$ such that:

- $a \in \operatorname{Int}\left(K_{0}\right)$;

- $d\left(K_{i}\right)<\varepsilon$ and $f\left(K_{i}\right) \subset \operatorname{Int}\left(K_{i+1}\right)$ for $0 \leq i \leq n+m-2$;

- $K_{i} \cap K_{j}=\varnothing$ for $i \neq j$;

- $K_{n} \subset V$ and $f\left(K_{n+m-1}\right) \subset V$.

Let $\rho>0$ be small enough that $m \rho^{\alpha}<1$ and $\rho<\beta$. For each $i$, we choose a compact $k_{i}$ with a non-empty interior included in $\operatorname{Int}\left(K_{i}\right)$ such that $d\left(k_{i}\right)<\rho$ and a point $b_{i}$ in $\operatorname{Int}\left(k_{i}\right)$.

For each $i$, let $\eta_{i}>0$ be such that the ball centred at $b_{i}$ of radius $\eta_{i}$ is included in $\operatorname{Int}\left(k_{i}\right)$. Then, we define a function $g$ in the following way:

For $0 \leq i \leq n+m-2$, we put $g=b_{i+1}$ on $k_{i}$ and $g=f$ on $\partial K_{i}$. On the one hand, $g$ takes its values in $K_{i+1}$ which is homeomorphic to $I^{N}$. On the other hand, $k_{i} \cup \partial K_{i}$ is a closed subset of $K_{i}$. So, according to the Tietze extension theorem (see [2, Corollary 1, p. 82]), $g$ has a continuous extension (which means that it still agrees with $f$ on $\partial K_{i}$ and with $b_{i+1}$ on $k_{i}$ ) defined on $K_{i}$ and taking its values in $K_{i+1}$. As $d\left(K_{i+1}\right)<\varepsilon, d[f(x), g(x)]<\varepsilon$ for each $x \in K_{i}$.

In the same way, we put $g=b_{n}$ on $k_{n+m-1}$ and $g=f$ on $\partial K_{n+m-1}$, and then we extend $g$ on $K_{n+m-1}$ into $\operatorname{Clos}(V)$.

We put $g=f$ outside $\bigcup_{i} K_{i}$.

So, as $g$ agrees with $f$ on the boundaries of the $K_{i}$, it follows that $g$ is 
continuous on $X$. As $d[f(x), g(x)]<\varepsilon$ for each $x \in X$ and the function $x \rightarrow d[f(x), g(x)]$ is continuous on the compact $X$, we have $d_{\infty}(f, g)<\varepsilon$.

Let $b=b_{0}$ and $\eta=\min \eta_{i}$. As $a$ and $b$ are both in $K_{0}, d(a, b)<\varepsilon$. Thus, we have defined $(g, b) \in C(X) \times X$ such that $\delta[(f, a),(g, b)]<\varepsilon$.

Let $(h, c) \in C(X) \times X$ be such that $\delta[(g, b),(h, c)]<\eta$, that is to say, $d_{\infty}(h, g)<\eta$ and $d(c, b)<\eta$.

If $x \in k_{i}$, then $g(x)=b_{i+1}$, so $d\left[b_{i+1}, h(x)\right]<\eta$; thus $d\left[b_{i+1}, h(x)\right]<$ $\eta_{i+1}$, and so $h(x) \in k_{i+1}$. Therefore, $h\left(k_{i}\right) \subset k_{i+1}$ for each $0 \leq i \leq n+m-2$. For the same reason, $h\left(k_{n+m-1}\right) \subset k_{n}$.

As $c \in k_{0}$, for $l \geq n$, the sequence $\left(h^{l}(c)\right)$ takes its values in $k_{n} \cup k_{n+1} \cup$ $\cdots \cup k_{n+m-1}$ which is a closed subset. Thus $\omega(h, c) \subset k_{n} \cup k_{n+1} \cup \cdots \cup k_{n+m-1}$. As $m \rho^{\alpha}<1$ and $\rho<\beta, d\left(k_{i}\right)<\beta$ for each $i$ and $\sum_{i} d\left(k_{i}\right)^{\alpha}<1$. Therefore, $(h, c)$ belongs to $E_{\alpha, \beta}$. So the ball of $C(X) \times X$ centred at $(g, b)$ of radius $\eta$ is included in $E_{\alpha, \beta}$.

So any ball of $C(X) \times X$ (centred at $(f, a)$ of radius $\varepsilon$ ) contains a point $(g, b)$ which is the centre of a ball (of radius $\eta$ ) included in $E_{\alpha, \beta}$, and the result follows.

Lemma 2. Let $U$ be an open set of $X$. Let $F_{U}$ be the set of those $(f, x) \in$ $C(X) \times X$ such that $\omega(f, x)$ either contains no point of $U$ or contains at least two points of $U$. Then $F_{U}$ contains an open, dense subset of $C(X) \times X$.

Proof. Let $(f, a) \in C(X) \times X$ and $\varepsilon>0$. We begin the construction as in the proof of Lemma 1. We obtain $n$ and $m$ as before. We shall distinguish two cases:

(1) For each $i$ such that $n \leq i \leq n+m-1, f^{i}(a) \in \operatorname{Ext}(U)$.

As $\operatorname{Ext}(U)$ is open, we can choose the $K_{i}$ for $0 \leq i \leq n+m-1$ such that $K_{i} \subset \operatorname{Ext}(U)$ for $n \leq i \leq n+m-1$. We choose any $\rho>0$ and then the $k_{i}$ and the $b_{i}$. As in the proof of Lemma 1, we obtain $(g, b) \in C(X) \times X$ and $\eta>0$ such that $\delta[(f, a),(g, b)]<\varepsilon$; and if $(h, c) \in C(X) \times X$ verifies $\delta[(g, b),(h, c)]<\eta$, then $\omega(h, c)$ has no point in $U$, so $(h, c)$ belongs to $F_{U}$.

(2) There is a $j$ such that $n \leq j \leq n+m-1$ and $f^{j}(a) \in \operatorname{Clos}(U)$.

We can choose the $K_{i}$ such that $K_{j} \cap U$ contains an open set $W$. Taking any $\rho>0$, we chose the $k_{i}$ and the $b_{i}$ as in the proof of Lemma 1 for $i<n$. Then, for each $i \geq n$, we choose $k_{i}$ and $k_{i}^{\prime}$ two disjoint compact subsets with non-empty interiors included in $\operatorname{Int}\left(K_{i}\right), b_{i}$ and $b_{i}^{\prime}$ as in the proof of Lemma 1 , and $k_{j}$ and $k_{j}^{\prime}$ in $W$. Then we modify the construction of the function $g$ in the following way:

- for $0 \leq i \leq n+m-2$, we put $g=b_{i+1}$ on $k_{i}$ and $g=b_{i+1}^{\prime}$ on $k_{i}^{\prime}$;

- we put $g=b_{n}^{\prime}$ on $k_{n+m-1}$ and $g=b_{n}$ on $k_{n+m-1}^{\prime}$.

As in the proof of Lemma 1, we obtain $(g, b) \in C(X) \times X$ and $\eta>0$ such that $\delta[(f, a),(g, b)]<\varepsilon$; and if $(h, c) \in C(X) \times X$ verifies $\delta[(g, b),(h, c)]$ $<\eta$, then $\omega(h, c)$ has at least two points in $U$, so $(h, c)$ belongs to $F_{U}$. The result follows.

\section{Results}

Theorem 1. For $(f, x)$ typical in $C(X) \times X, \omega(f, x)$ is a perfect set of Hausdorff dimension zero. 
Proof. Let $E$ be the intersection of the $E_{1 / n, 1 / m}$ for $n, m \geq 1$ and $F_{U_{p}}$, $\left(U_{p}\right)_{p}$ being a countable base of open subsets of $X$. As the set of the triples $(n, m, p)$ is countable and according to Lemmas 1 and $2, E$ is residual.

Let $(f, x) \in E$. For each $(\alpha, \beta),(f, x) \in E_{\alpha, \beta}$; thus, $\omega(f, x)$ can be covered by a finite number of sets $\left(k_{i}\right)_{i}$ satisfying: for each $i, d\left(k_{i}\right)<\beta$ and $\sum_{i} d\left(k_{i}\right)^{\alpha}<1$. Therefore, according to the definition of the Hausdorff dimension (see [2]), $\omega(f, x)$ is of Hausdorff dimension zero.

For each open subset $U$ of $X,(f, x) \in F_{U}$; thus either $\omega(f, x)$ has no point in $U$ or at least two. Therefore, $\omega(f, x)$ does not contain an isolated point. As $\omega(f, x)$ is compact, $\omega(f, x)$ is a perfect set.

So we have found $E$, a residual subset of $C(X) \times X$, such that if $(f, x) \in E$, then $\omega(f, x)$ is a perfect set of Hausdorff dimension zero. That is to say, for $(f, x)$ typical in $C(X) \times X, \omega(f, x)$ is a perfect set of Hausdorff dimension zero.

Theorem 2. If $f$ is typical in $C(X)$, then, for $x$ typical in $X, \omega(f, x)$ is a perfect set of Hausdorff dimension zero.

Proof. $C(X)$ and $X$ are two complete metric spaces and $X$ is second countable. Let $E$ be the residual subset of $C(X) \times X$ of the proof of Theorem 1 . According to the Kuratowski-Ulam theorem (see [4, p. 56]), there is a residual subset $F$ of $C(X)$ such that for each $f \in F$ the section of $E$ by $f$ (i.e., $\left.E_{f}=\{x \in X ;(f, x) \in E\}\right)$ is a residual subset of $X$. If $f \in F$ and $x \in E_{f}$, then $(f, x) \in E$, so $\omega(f, x)$ is a perfect set of Hausdorff dimension zero. That is to say, if $f$ is typical in $C(X)$, then, for $x$ typical in $X, \omega(f, x)$ is a perfect set of Hausdorff dimension zero.

Theorem 3. The function $\omega$ is continuous at the typical point $(f, x)$ of $C(X) \times$ $X$.

Proof. Let $\alpha>0$ and $G_{\alpha}$ be the set of those $(f, x) \in C(X) \times X$ such that there is a neighbourhood $U$ of $(f, x)$ on which $d_{K}\left[\omega(f, x), \omega\left(f^{\prime}, x^{\prime}\right)\right]<\alpha$ for each $\left(f^{\prime}, x^{\prime}\right) \in U$.

Let $(f, a) \in C(X) \times X$ and $\varepsilon>0$. We begin as in the proof of Lemma 1 . We obtain the $K_{i}$ and then choose $\rho>0$ such that $m \rho<\alpha$. Then we obtain the $k_{i}$ such that $d\left(k_{n}\right)+d\left(k_{n+1}\right)+\cdots+d\left(k_{n+m-1}\right)<\alpha$. Then we construct $(g, b) \in C(X) \times X$ and $\eta>0$ as in the proof of Lemma 1 . Let $B$ be the open ball of $C(X) \times X$ centred at $(g, b)$ of radius $\eta$. If $(h, c) \in B$, then $\omega(h, c) \subset k_{n} \cup k_{n+1} \cup \cdots \cup k_{n+m-1}$ and $\omega(h, c) \cap k_{i} \neq \varnothing$ for $n \leq i \leq n+m-1$. Thus, if $(h, c)$ and $\left(h^{\prime}, c^{\prime}\right)$ belong to $B$, then $d_{K}\left[\omega(h, c), \omega\left(h^{\prime}, c^{\prime}\right)\right]<\alpha$.

Let $(h, c) \in B$. Since $B$ is an open set, there is an open neighbourhood $V$ of $(h, c)$ contained in $B$. If $\left(h^{\prime}, c^{\prime}\right) \in V$, then $d_{K}\left[\omega(h, c), \omega\left(h^{\prime}, c^{\prime}\right)\right]<\alpha$. Hence $(h, c) \in G_{\alpha}$. As in the proof of Lemma 1, it follows that $G_{\alpha}$ contains a dense, open subset of $C(X) \times X$.

The intersection $G$ of the $G_{1 / n}$ for $n \geq 1$ is residual. Let $(f, x) \in G$. Let $\alpha>0$; then $(f, x) \in G_{\alpha}$, so there is a neighbourhood $U$ of $(f, x)$ such that $d_{K}\left[\omega(f, x), \omega\left(f^{\prime}, x^{\prime}\right)\right]<\alpha$ for each $\left(f^{\prime}, x^{\prime}\right) \in U$. This means that $\omega$ is continuous at the point $(f, x)$. Therefore, $\omega$ is continuous at any point of $G$. The result follows.

Theorem 4. If $f$ is typical in $C(X)$, the function $x \rightarrow \omega(f, x)$ is continuous at the typical point $x$ of $X$. 
Proof. As in the proof of Theorem 2, the Kuratowski-Ulam theorem allows us to deduce this result from the previous one.

\section{REMARKS}

Remark 1. According to Mandelbrot (see [3, p. 15]), a fractal is a set for which the two dimensions used in this paper (and in [2]) are distinct. Hence the typical limit set is not a fractal.

Remark 2. In chaotic sequences, the limit set has a sensitive dependence on the initial condition. As for $f$ typical in $C(X)$, the function $x \rightarrow \omega(f, x)$ is continuous at the typical point of $X$; it follows that the sequence $\left(f^{n}(x)\right)$ is not chaotic.

\section{REFERENCES}

1. S. J. Agronsky, A. M. Bruckner, and M. Laczkovich, Dynamics of typical continuous functions, J. London Math. Soc. (3) 131 (1989), 227-243.

2. W. Hurewicz and H. Wallman, Dimension theory, Princeton Univ. Press, Princeton, NJ, 1948.

3. B. Mandelbrot, Fractals: Form, chance and dimension, Freeman, San Francisco, 1977.

4. J. C. Oxtoby, Measure and category, Springer-Verlag, New York, 1980.

5. J. Nagata, Modern general topology, North-Holland, New York, 1974.

Ecole Normale Superieure de Cachan, 61 Avenue du President Wilson, 94235 Cachan Cedex, France

Current address: 13 Rue Letellier, 75015 Paris, France 